\title{
Microsatellite Markers to Complement Distinctness, Uniformity, Stability Testing of Brassica chinensis (Xiao Baicai) Varieties
}

\author{
G.R. Yim ${ }^{1,2}$, D.R. Ding ${ }^{1}$, C.P. Wang ${ }^{3}$, W.H. Leong ${ }^{3}$ and Y. Hong ${ }^{1,2, *}$ \\ ${ }^{1}$ Temasek Life Sciences Laboratory, 1 Research Link, The National University of Singapore, Singapore 117604; ${ }^{2}$ School \\ of Biological Sciences, Nanyang Technological University, Singapore 637551 and ${ }^{3}$ Horticulture Branch, Agri-food and \\ Veterinary Authority of Singapore, Sembawang Research Station, Singapore 769194
}

\begin{abstract}
Brassica chinensis varieties are mostly consumed as vegetables. In order to assess utility of microsatellite markers for testing distinctness, uniformity and stability of $B$. chinensis varieties, we used nine polymorphic microsatellite marker loci (with a total of 131 alleles) to evaluate four open pollinated and four hybrid varieties of B. chinensis. Each variety was represented by 48 randomly sampled individuals from a population of 150 . Morphological evaluations were also conducted to the same samples. Compared with morphological evaluations, the microsatellite analysis provided a higher degree of separation and reflected greater uniformity for the three hybrid varieties by cluster analysis, Principal Coordinates Analysis (PCA) and assignment test. Average pair-wise Jaccard similarities and statistical tests by AMOVA (analysis of molecular variance) also suggested higher levels of intra-variety uniformity. It was also noted that there was no significant correlation between the genetic and morphological distance matrices (Mantel test). We conclude that microsatellite genotyping can be a valuable tool to complement morphological assessment of Brassica vegetable varieties for distinctiveness and uniformity.
\end{abstract}

Key Words: SSR, microsatellite, genetic profiling, DUS, Brassica vegetable, Xiao Baicai.

\section{INTRODUCTION}

In an international and intergovernmental effort to protect new varieties of plants as intellectual property rights, the International Union for the Protection of New Varieties of Plants (UPOV) was established in 1961. According to UPOV regulations, a 'new' plant variety must be Novel, Distinct, Uniform and Stable (DUS) in order for a plant breeder to obtain Plant Breeder Rights. Singapore became a member of UPOV on 30th July 2004. In Singapore, the Agrifood Veterinary Authority of Singapore (AVA) conducts technical evaluations based on DUS requirements before breeder rights are awarded by the Intellectual Property Office of Singapore. Two Brassica vegetables varieties, $B$. chinensis L. (Baicai, Xiao baicai) and B. chinensis var. parachinensis (Caixin) are currently protected in Singapore.

Good genetic compatibility among Brassica species enables them to undergo intraspecific, interspecific and even intergeneric crosses to create hybrid varieties [1]. Hybrid vigor in hybrid varieties has been widely exploited in $B$. napus and B. juncea as oil crops. B. chinensis varieties are mostly used as vegetables. They are known to exhibit inbreeding depression - decreased vigor for plants derived from self pollination. Most $B$. chinensis vegetable varieties belong to Heirloom varieties whose seeds are collected from open-pollinated populations while some $B$. chinensis hybrids are available in the market. Open pollinated seeds are poten-

*Address correspondence to this author at the Temasek Life Sciences Laboratory, 1 Research Link, The National University of Singapore, Singapore 117604; Tel: (65)-68727095; Fax: (65)-68727007; E-mail: hongy @tll.org.sg tially heterozygous and pose a special difficulty in technical evaluation based on DUS requirements. On the other hand, morphology of plants can be influenced by various environmental conditions $[2,3]$. Phenotypes are not strictly comparable unless plants are grown under the exact same conditions. As such, there are many complications in DUS evaluation - like the cost and space required for comprehensive evaluation.

Considering cost and effort involved in morphologybased evaluation, alternatives to morphological assessment have been widely explored. There is strong interest to use molecular markers as complementary methods for new variety description. Genetic markers are more stable and are largely unaffected by changes of environment or different plant developmental stages. They can provide a larger degree of polymorphism in contrast with morphological descriptors. DNA molecular markers investigated for application in DUS testing include Amplified Fragment Length Polymorphism (AFLP), Random Amplification of Polymorphic DNA (RAPD) and Simple Sequence Repeats (SSR).

SSR or microsatellites are short DNA repeat units of two to six base pairs abundantly interspersed throughout eukaryotic genomes [4]. They occur once every 30-60 kb [5] and concentrate in the non-coding regions of genomic DNA. They probably do not have any role in gene expression. It was proposed [6] that microsatellites arisen from slippage replication and unequal crossover. Microsatellite loci are highly polymorphic, specific and informative, making them suitable as genetic markers. These loci can be amplified through primers localized at stable and conserved flanking 
regions. They display Mendelian codominant inheritance and provide reproducible genotyping. Microsatellites have been tested to complement DUS testing in B. napus L. [7], aromatic rice [8], wheat and tomato [9]. They were found to be able to separate varieties and establish levels of uniformity and distinctness in all the reports.

Previously, we identified nine microsatellite markers suitable for differentiating Brassica vegetable varieties. In this study, we examined the applicability of these microsatellite markers to complement morphological analysis in DUS evaluation of eight $B$. chinensis varieties. Their ability to determine the genetic diversity within and between varieties was also assessed.

\section{MATERIALS AND METHODS}

\section{Plant Materials}

Seeds of the $B$. chinensis vegetables were obtained from Mainland and Taiwan of China and Japan. C1, S162, S201 and S222 were labeled as hybrid seeds whereas S148, S188, S194 and S200 were presumed open pollinated ones.

The eight varieties of $B$. chinensis were planted on soil in a growing tunnel house at the AVA Horticulture Branch, Sembawang, Singapore. Each variety was grown in three replicate beds of 50 seedlings each. Plant beds were allocated randomly. Plants were harvested 30 days after seed sowing and 16 individual plants from each replicate's diagonal axis were selected for morphological and genetic analysis. A total of 384 plants (48 per variety) were harvested.

\section{Extraction of Plant DNA}

Leaf samples were collected from every harvested plant for genotyping. About 50mg of fresh leaf tissue from each plant was collected for plant DNA isolation using the QIAGEN Dneasy 96 Plant Kit (QIAGEN GmbH, Strasee, Germany). Plant tissue was disrupted using the Mixer Mill MM 300 (Retsch GmbH, Haan, Germany) with the Mixer Mill Adapter set and Tungsten Carbide beads (both from QIAGEN GmbH, Strasee, Germany). Extraction procedure was based on the manufacturer's protocol.

\section{Microsatellite Genotyping}

Genomic DNA samples were diluted in TE $(0.1)(10 \mathrm{mM}$ Tris $\mathrm{pH} 8.0$ and $0.1 \mathrm{mM}$ EDTA) to $10 \mathrm{ng} / \mu \mathrm{l}$ before amplification by polymerase chain reaction (PCR). The PCR mixture, in a total volume of $20 \mu \mathrm{L}$, contained $2 \mu \mathrm{L}$ of genomic DNA template, 1 x PCR buffer, $2.125 \mathrm{mM} \mathrm{MgCl}_{2}$, I U of Taq DNA polymerase (QIAGEN GmbH, Strasee, Germany), $0.5 \mu \mathrm{M}$ of each primer (1st Base Pte Ltd, Singapore) and $0.2 \mathrm{mM}$ of each dNTP (iDNA Biotechnology Pte Ltd, Singapore). PCR was carried out using PTC-100 Thermal Cycler machines (MJ Research, Inc. Waltham, USA) with the following profile: Hot start of $94^{\circ} \mathrm{C}$ for 5 minutes, followed by 36 cycles of an initial denaturation step $\left(94^{\circ} \mathrm{C}, 40\right.$ seconds), annealing step $\left(51^{\circ} \mathrm{C}, 1\right.$ minute) and elongation step $\left(72^{\circ} \mathrm{C}\right.$, 1.5 minutes) followed by a final step of $72^{\circ} \mathrm{C}$ for 7 minutes. The PCR products were mixed with GeneScan -500 ROX size standards and separated using the ABI 3730XL sequencer. The fragment sizes were analyzed with Applied Biosystems GeneMapper version 3.7 (Applied Biosystems, Foster City, USA).

\section{Morphological Analysis}

Plant morphological characters were analyzed according to AVA guidelines (Table 1) for Brassica vegetables. The Singapore guideline adapted other UPOV guidelines on Brassica species with some modifications. Outliers in the 48 plants for each variety were identified and recorded.

\section{Data Analysis}

Microsatellite allele distribution data obtained from Genemapper 3.7 was first converted into suitable formats for downstream genetic and statistical analysis with CONVERT 1.31 [10]. Genetic Data Analysis (GDA 1.1) [11] was used for standard descriptive statistics. F-statistical analysis was conducted to the same set of data with FSTAT 2.9.3 [12]. An AFLP Excel Macro [13] was used to convert the allele size data from Genemapper 3.7 into binary form, to indicate the presence (1) or absence (0) of alleles. Similarly, morphological characters were converted to positive (1) or negative (0) results for each character outcome. NTSYSpc 2.11S (Exeter software, Setauket, NY) was then employed for calculation of Jaccard similarity coefficients for both genetic and morphological data, clustering by Unweighted Pair-Group Method using Arithmetic averages (UPGMA) and Mantel tests for the correlation between the genetic and morphological matrices. Average intra-variety similarities and standard deviations were subsequently calculated. GenAlEx 6 [14] was employed to compute the average probability of identity (PI) for each locus, $\Phi_{\mathrm{PT}}$ values via Analysis of Molecular Variance (AMOVA) (treating each variety as one population), Principal coordinates analysis (PCA) and assignment test.

\section{RESULTS}

\section{Informativeness of the Nine Microsatellite Loci}

Table 2 presents the genetic diversity measures of the nine microsatellite loci in the population of 384 plants of eight varieties. A total of 131 microsatellite alleles were identified. The microsatellite loci gave a mean expected and observed heterozygosity of 0.61 and 0.44 respectively. The average inbreeding coefficient is 0.283 , indicating heterozygous excess. The combined PI for the nine loci is $3.9359 \mathrm{x}$ $10^{-9}$, i.e. one out of 3.9 billion unrelated plants will share an identical SSR profile by chance as any one of the 384 plants analyzed.

\section{Determination of Distinctness and Uniformity by SSR Analysis}

The 131 microsatellite alleles separate the three $B$. chinensis hybrid varieties C1, S162 and S201 from the other 5 varieties, as shown in the dendrogram (Fig. 1). Several plants within these varieties share identical SSR profiles. From the dendrogram, C1 plants (except for the outlier C110) share at least $76 \%$ similarity. S162 (excluding the outlier S164-14) and S201 plants show at least 62\% and 83\% similarity respectively. On the contrary, plants of the other 5 varieties do not form distinct clusters, suggesting them genetically neither distinct nor uniform. From the cluster analysis, division between the genetically distinct and uniform varieties with those non-distinct and non-uniform varieties is unambiguous. 
Table 1. Morphological Traits and Characters of B. chinensis Varieties Used for DUS Testing at AVA

\begin{tabular}{|c|c|c|c|c|c|}
\hline & Morphological Trait & \multicolumn{4}{|c|}{ Characters } \\
\hline 1 & Seed size & a. Small & b. Medium & c. Large & \\
\hline 2 & Intensity of brown color of seed & a. Light & b. Medium & c. Dark & \\
\hline 3 & Attitude of plant & a. Erect & b. Semi-erect & c. Horizontal & \\
\hline 4 & Position of growth point in relation to top of plant & a. Same level & b. Slightly below & c. Deeply below & \\
\hline 5 & Plant length without root & a. Very Short & b. Short & c. Medium & \\
\hline 6 & Plant width & a. Narrow & b. Medium & c. Broad & \\
\hline 7 & Length of Cotyledonous node to growing point & a. Very Short & b. Short & c. Medium & \\
\hline 8 & Leaf arrangement & a. Rosulate & b. Alternate & & \\
\hline 9 & Number of leaves & a. Few & b. Medium & c. More & \\
\hline 10 & Blistering of blade surface & a. Absent/ Very weak & b. Weak & c. Medium & d. Strong \\
\hline 11 & Width of largest leaf & a. Narrow & b. Medium & c. Broad & \\
\hline 12 & Length of largest leaf (excluding petiole) & a. Short & b. Medium & c. Long & \\
\hline 13 & Shape of leaf blade & a. Narrow elliptic & b. Narrow elliptic to elliptic & c. Elliptic & d. Broad elliptic \\
\hline 14 & Intensity of green color of fully developed leaf & a. Light & b. Medium & c. Dark & \\
\hline 15 & Hairs on leaf surface & a. Absent & b. Weak & c. Medium & \\
\hline 16 & Shape of leaf apex & a. Mucronate & b. Obtuse & c. Emarginate & \\
\hline 17 & Shape of leaf base & a. Cuneate & b. Obtuse & c. Obtuse with lobe & \\
\hline 18 & Leaf blade margin & a. Sinuate & b. Entire & & \\
\hline 19 & Petiole length (below leaf base to leaf node) & a. Short & b. Medium & c. Long & \\
\hline 20 & Petiole width (broadest area on base) & a. Narrow & b. Medium & c. Broad & \\
\hline 21 & Intensity of green color of Petiole & a. White & b. Light & c. Medium & d. Dark \\
\hline
\end{tabular}

The measure of average pairwise Jaccard similarity gives a better depiction of intra-variety uniformity. Fig. (2) shows that while C1, S162 and S201 plants share at least 70\% similarity, the intra-variety uniformity of the other five varieties fall below $43 \%$.

Principal coordinates analysis (PCA) for the genetic data (Fig. 3) likewise separated C1, S162 and S201 clearly. The rest of the varieties were less distinguishable from each other.

Assignment tests (Table 3) indicate that a total of 353 plants could be assigned to their respective varieties. All plants from the three hybrid varieties were allocated correctly.

\section{Determination of Distinctness and Uniformity by Mor- phological Analysis}

Morphological observation identified at least one outlier among the 48 tested plants for every variety, hence it cannot conclusively determine the uniformity of any variety. Plants for S200 were highly variable in morphology. With regards to distinctiveness, only $\mathrm{C} 1$ was unambiguously distinct; while no consensus was reached for the other varieties. The combination of morphological characters for cluster analysis showed that most of the 48 plants from each variety form distinct clusters, with the exception of S200 (Fig. 4). S162, S194 and S222 have only one plant deviant from their respective clusters. S162-14, the morphological outlier of the variety, is also the genetic outlier. It was probably due to a mix-up of seeds. The other genetic outlier C1-10, however, does not display distinctiveness in morphology. With respect to their morphological traits, plants of the C1, S148, S188 and S201 varieties share at least 63\%, 63\%, 52\% and 53\% similarity respectively. The main cluster of S162 show at least $70 \%$ similarity. Although cluster analysis using morphological traits can differentiate among varieties, it does not give clear indication whether a variety is uniform.

This lack of distinction can be seen from the average pair-wise Jaccard similarities for morphological traits (Fig. 5). In contrast with Fig. (2), the intra variety morphology similarity for the three hybrid varieties is not as apparent as genetic similarity suggests.

Morphological PCA (Fig. 6), in contrast with PCA for genetic data, do not reflect the genetic distinction of the three hybrid varieties. None of the eight varieties analyzed is grouped into individual clusters. 
Table 2. Information on the 9 Microsatellite Markers in the Population of 384 Plants of 8 B. chinenesis Varieties

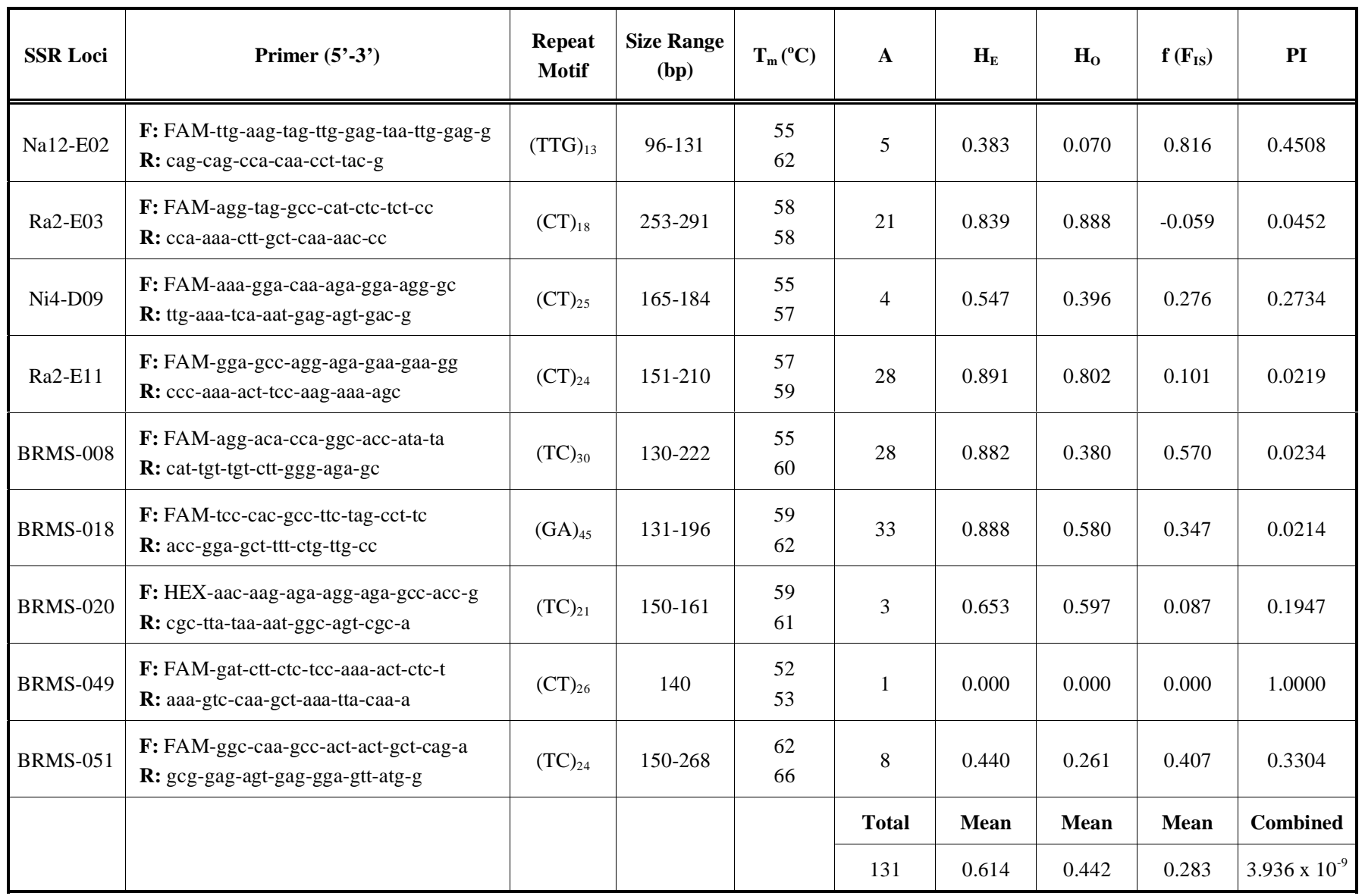

Legend: $\mathrm{A}=$ number of Alleles per locus; $\mathrm{H}_{\mathrm{E}} / \mathrm{H}_{\mathrm{O}}=$ Expected/ Observed Heterozygosity; $\mathrm{f}=$ inbreeding coefficient; $\mathrm{PI}=$ Probability of Identity.

Table 3. Population Assignment Test Summary

\begin{tabular}{|c|c|c|}
\hline Population & Self Population & Other Populations \\
\hline \hline C1 & 48 & \\
\hline S148 & 47 & 1 \\
\hline S162 & 48 & 8 \\
\hline S188 & 40 & 4 \\
\hline S194 & 44 & 11 \\
\hline S200 & 37 & \\
\hline S201 & 48 & 7 \\
\hline S222 & 41 & 31 \\
\hline Total & 353 & \\
\hline
\end{tabular}

\section{Analysis of Molecular Variance}

Analysis of Molecular Variance (AMOVA) is used to evaluate structure of diversity (Table 4). The Sum of Squares within population (SSWP) reflects intra-variety diversity. For genetic data, SSWP for the three hybrids C1, S162 and S201 are much lower (all <134) than the other five (all
$>260$ ). For morphological data, SSWP for C1, S162 and S201 varieties are less but not much different from those for the other five. Estimation of $\Phi_{\mathrm{PT}}$ by AMOVA gives an indication of variation among varieties. For both genetic and morphological data, $\Phi_{\mathrm{PT}}$ values are statistically significant $(\mathrm{p}$ $=0.001)$, indicating more contribution by inter-variety variation.

\section{Genetic-Morphological Correlation}

The correlation between the genetic and morphological distance matrices is calculated to determine the relationship between the two methods of analysis. The two-way Mantel test (Fig. 7) gives a matrix correlation coefficient (r) of 0.415 , suggesting poor correlation between the two matrices. An additional test (data not shown) for Pairwise Population genetic and morphological matrices calculated by Nei genetic distance, gives a correlation coefficient of 0.074 (normalized), with $42.7 \%$ significance by random permutation, in agreement with poor correlation suggested from the Mantel test.

\section{DISCUSSION}

\section{Suitability of the Nine Microsatellite Loci}

The level of genetic variation with respect to a locus is proportional to the heterozygosity. In this experiment, each 


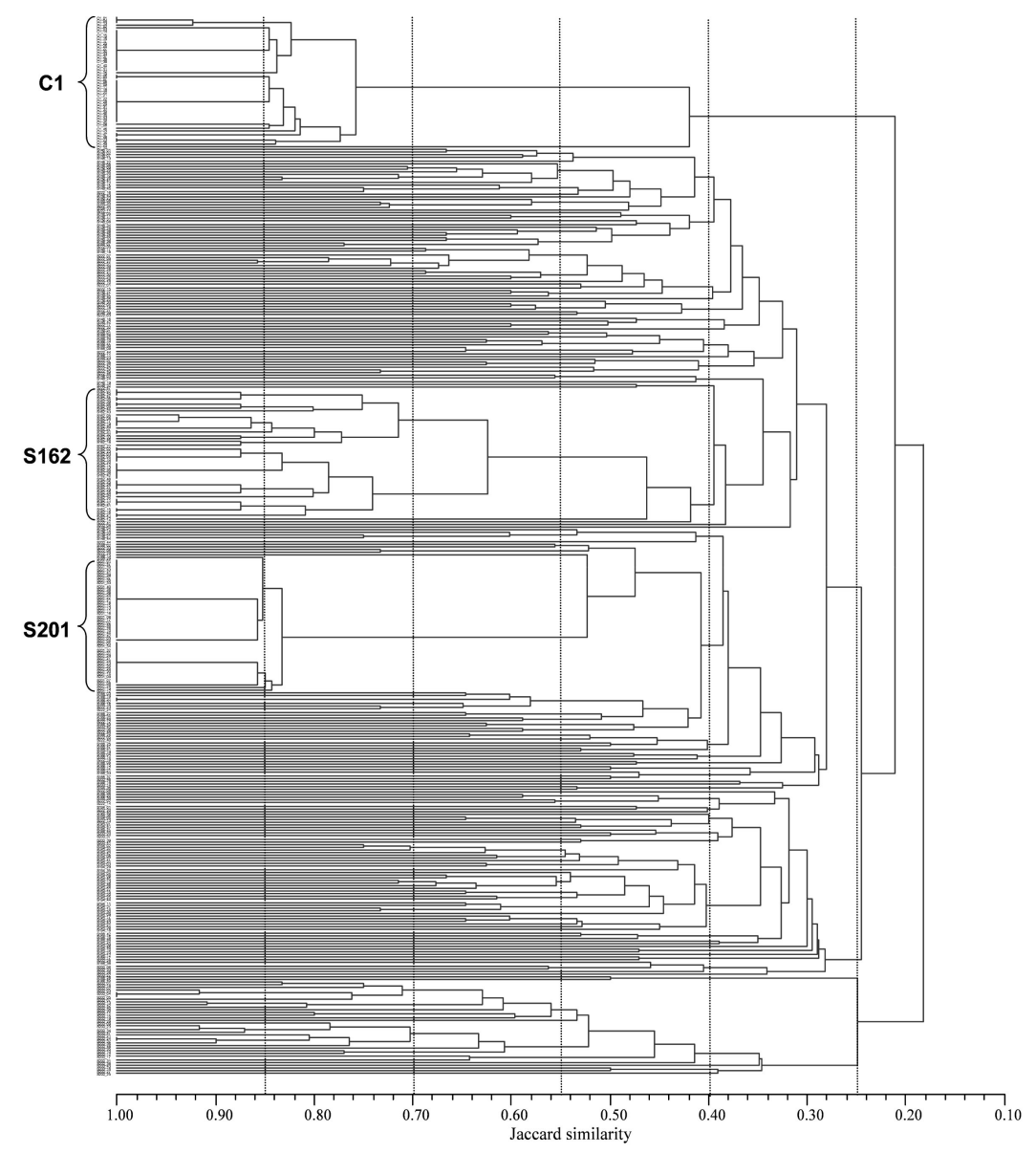

Fig. (1). Cluster analysis of individual plants based on 9 microsatellite loci.

locus has an average of 14.5 alleles. The nine microsatellite loci (together with 131 alleles) are highly polymorphic and display different degrees of genetic variations, with the exception of BRMS-049, the only one that is fully homozygous. Despite its homozygosity among the $8 \mathrm{~B}$. chinensis varieties, BRMS-049 can differentiate between $B$. oleracea and $B$. chinensis with great reliability as shown in our previous work (data not shown). This locus serves as a positive control of PCR amplification.

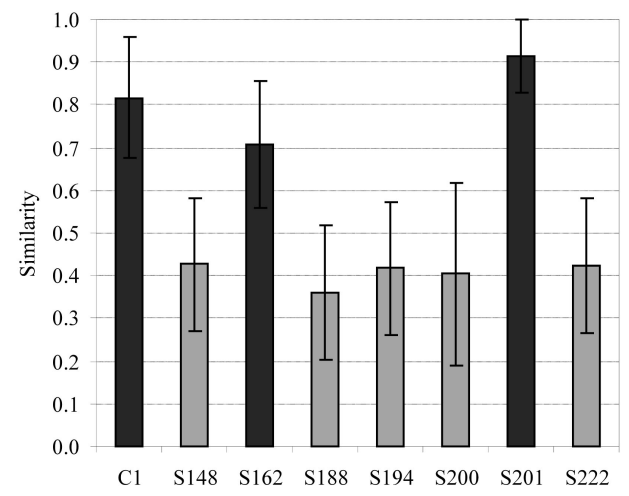

Fig. (2). Average pairwise intra-variety genetic similarity in the eight varieties. Standard derivations (SD) are indicated by the lines above and below columns.
The nine microsatellite loci give an average $\mathrm{F}_{\mathrm{IS}}$ (inbreeding coefficient) of 0.283 . Probability of Identity (PI) is the chance that a set of alleles exists in two unrelated individuals [15]. The PI for the combination of nine microsatellite markers is $3.9359 \times 10^{-9}$. This means that, in B. chinensis plants, there is only the chance of one in 3.9 billion that two unrelated individuals would share an identical allele profile. It proves the reliability of the loci when used together as genetic markers for $B$. chinensis vegetables.

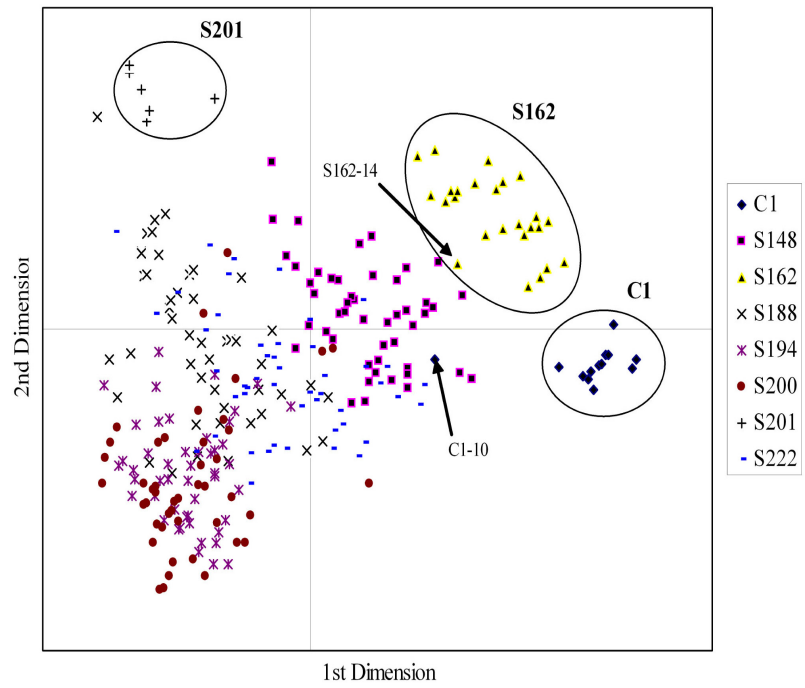

Fig (3). Principal Coordinates Analysis (PCA) for genetic data of the eight $B$. chinensis vegetables. 


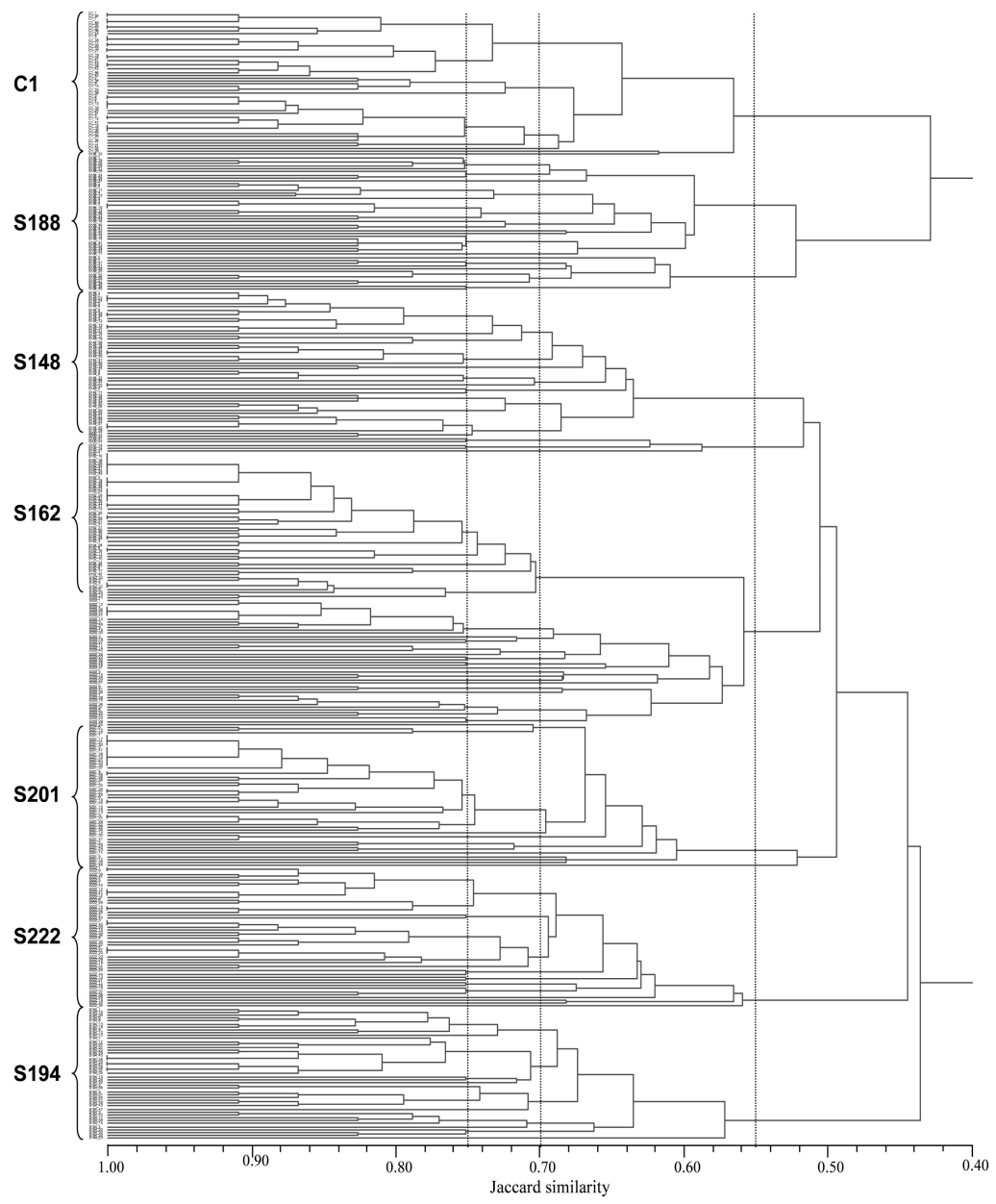

Fig. (4). Cluster analysis of individual plants based on morphological traits.

As a whole, the nine microsatellites are highly polymorphic. which makes them suitable as tools for genotyping $B$. chinensis varieties.

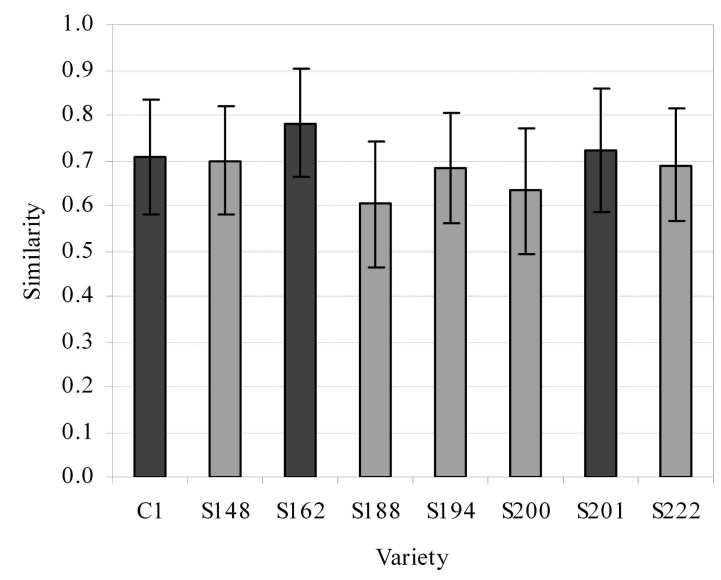

Fig. (5). Average pairwise intra-variety morphological similarity in the eight $B$. chinensis vegetables.

\section{Determination of Distinctness and Uniformity}

In this work, the nine microsatellite markers were evaluated for ability to determine the distinctness of Xiao Baicai varieties and the ability to assess uniformity. If a variety was not genetically distinct, the 48 samples would be unable to form a cluster. Quality of clustering would also reflect uniformity. In this study, we found out that only three hybrid

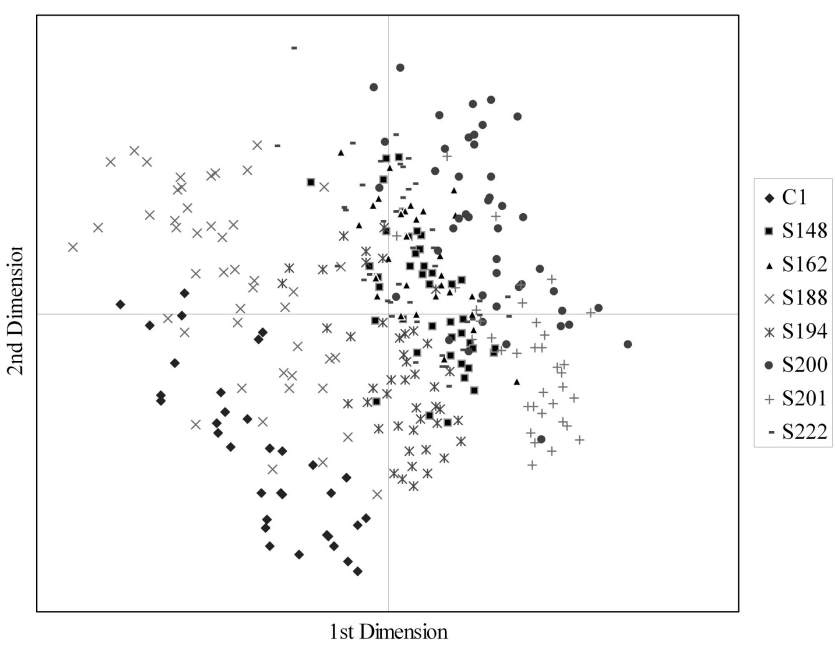

Fig. (6). Principal Coordinates Analysis (PCA) of morphology data for the eight $B$. chinensis vegetables.

varieties (C1, S162 and S201) could form distinct cluster. PCA analysis could similarly separate the three varieties 
Table 4. Analysis of Genetic and Morphology Variance within Populations

\begin{tabular}{|c|c|c|c|c|c|c|c|c|}
\hline Variety & C1 & S148 & S162 & S188 & S194 & S200 & S201 & S222 \\
\hline \hline Number of samples & 48 & 48 & 48 & 48 & 48 & 48 & 48 & 48 \\
\hline SSWP (genetic)* & 65.0 & 261.0 & 133.2 & 323.0 & 264.9 & 264.0 & 29.6 & 267.1 \\
\hline SSWP (morphological)* & 90.9 & 93.7 & 65.7 & 132.3 & 99.9 & 120.3 & 86.9 & 98.1 \\
\hline
\end{tabular}

* All $\Phi_{\mathrm{PT}}$ are statistically significant at $\mathrm{p}<0.001$.

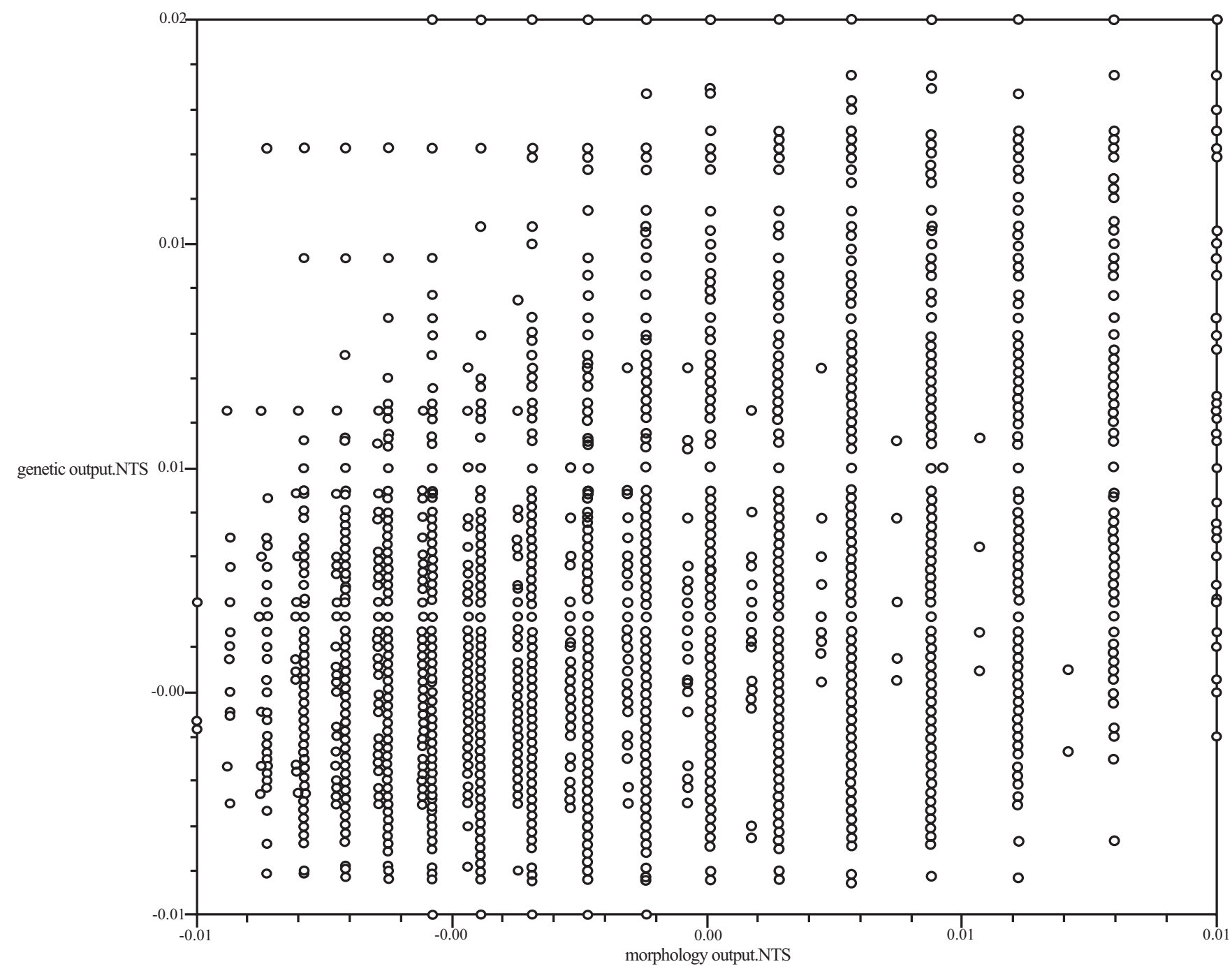

Fig. (7). Mantel test between genetic and morphological distance matrices for the eight B. chinensis vegetables.

distinctly from others. Assignment test based on genetic profile was $100 \%$ for the same three varieties. All these point to the conclusion that the three hybrids are distinct. In comparison with morphological data, seven out of the eight varieties can be clustered but PCA analysis test failed to separate any single variety.

In order to determine intra-variety uniformity quantitatively, we calculated the average of pair-wise Jaccard similarities between individual plants of each variety. C1, S162 and S201 (>70\%) show a distinctly higher intra-variety simi- larity than the other varieties $(<43 \%)$. In contrast, the average pairwise similarities for the morphological traits are unable to achieve this level of differentiation.

AMOVA test was also conducted for all the varieties. $\Phi$ statistics were significant $(\mathrm{p}<0.001)$ for genetic data and also morphological data, suggesting the statistically significant contribution by inter variety variance. SSWP represents intra variety variance. Comparison of SSWP for the eight varieties indicated that the same three hybrid varieties had SSWP values (all lower than 134) significantly lower than those for 
other varieties (all above 260). Both approaches agree to the distinctly higher level of genetic uniformity in these three hybrid varieties. In comparison, morphological data gave lower SSWP values for the three hybrids, but they were not as distinct from the other varieties as genetic data suggested. We conclude that with AMOVA test, genetic data is more defining than morphological data for uniformity.

\section{Genetic and Morphological Correlation}

Although morphological characters and traits set by the AVA serve to translate visual observation into quantitative and qualitative data, some other important characters like pod and flower characteristics are not included for the consideration of feasibility. These 21 traits with 64 characters may not be sufficient to reach a definite conclusion. This is reflected in the failure of PCA analysis (based on morphological data) to separate any variety conclusively. In contrast, nine microsatellite loci with 131 alleles reliably differentiated the individual plants. Our work found poor geneticmorphological correlation $(\mathrm{r}=0.41474)$. The lack of correlation between SSR and morphological data $(r=0.098)$ was also reported in B. napus L. varieties [7]. Other genetic methods like RAPD in cucumber gave poor correlation as well $(\mathrm{r}=0.65)$ [16]. This is not totally surprising since microsatellite loci are not quantitative trait loci. They are found mostly in non-coding regions and may not be directly involved in gene expression; whereas phenotypic traits are largely affected by gene expression. The two methods could be independent of each other.

\section{Applicability of SSR Markers to Complement DUS Evaluation}

A variety has to be morphologically novel, distinct, uniform and stable to qualify for plant variety protection. SSR analysis can add depth and resolution to morphological analysis. In the light of discriminatory power presented in this paper, we suggest that these nine microsatellite loci are suitable for B. chinensis varieties in distinctiveness and uniformity assessment. With the three hybrid varieties C1, S162 and S201 as references, we can employ the following criteria to judge distinctiveness: clear clustering of random samples, non-ambiguous separation by PCA analysis and 100\% success in assignment test. As to uniformity, we can set a Jaccard similarity (pair-wise average within a variety) value of 0.7 as the cut-off point. Also, SSWP by AMOVA test should be less than 70 . With the advent of fluorescent multiplexing PCR and automated detection and sizing, SSR genotyping will save monetary and labor costs, and become a valuable part to DUS evaluation. It is, however, not a replacement for morphological evaluation due to the poor correlation of microsatellite genetic analysis with morphological variation at the individual plant level.

\section{ACKNOWLEDGMENT}

This project was supported by a research grant from Agri-food and Veterinary Authority of Singapore and Final Year Project of Nanyang Technological University. We thank L.T. LAM-CHAN, B.L. POH and K.K. TAN for technical help and critical discussions.

\section{REFERENCES}

[1] Myers JR. Outcrossing potential for brassica species and implications for vegetable crucifer seed crops of growing oilseed Brassicas in the willamette valley. Oregon State University Extension Service Special Report 10642006.

[2] Cipollini DF. Costs to flowering of the production of a mechanically hardened phenotype in Brassica napus L. Int J Plant Sci 1999; 160: 735-41.

[3] Omer LS, Horvath SM. Developmental changes in anatomy, morphology and biochemistry of Layia platyglossa exposed to elevated carbon dioxide. Am J Bot 1984; 71: 693-99.

[4] Miesfeld R, Krystal M, Amheim N. A member of a new repeated sequence family which is conserved throughout eucaryotic evolution is found between the human delta and beta globin genes. Nucleic Acids Res 1981; 9: 5931-48.

[5] Weber JL, May PE. Abundant class of human DNA polymorphisms which can be typed using the polymerase chain reaction. Am J Hum Genet 1989; 44: 388-96.

[6] Tautz D, Renz M. Simple sequences are ubiquitous repetitive components of eukaryotic genomes. Nucleic Acids Res 1984; 12 : 4127-38.

[7] Tommasini L, Batley J, Arnold GM, et al. The development of multiplex simple sequence repeat (SSR) markers to complement distinctness, uniformity and stability testing of rape (Brassica napus L.) varieties. Theor Appl Genet 2003; 106: 1091-101.

[8] Singh RK, Sharma RK, Singh AK, et al. Suitability of mapped sequence tagged microsatellite site markers for establishing distinctness, uniformity and stability in aromatic rice. Euphytica 2004; 135: $135-43$.

[9] Cooke RJ, Bredemeijer GMM, Ganal MW, et al. Assessment of the uniformity of wheat and tomato varieties at DNA microsatellite loci. Euphytica 2003; 132: 331-41.

[10] Glaubitz JC. convert: A user-friendly program to reformat diploid genotypic data for commonly used population genetic software packages. Mol Ecol Notes 2004; 4: 309-10.

[11] Lewis PO, Zaykin D. Genetic Data Analysis: Computer program for the analysis of allelic data. Version 1.0 (d16c) 2007. Available from: http://www.eeb.uconn.edu/people/plewis/software.php

[12] Goudet J. FSTAT, a program to estimate and test gene diversities and fixation indices (version 2.9.3) 2007. Available from: http://www.unil.ch/izea/softwares/fstat.html

[13] Rinehart TA. AFLP Analysis using genemapper software and an excel macro that aligns and converts output to binary. Biotechniques 2004; 37: 186-88.

[14] Peakall R, Smouse PE. genalex 6: genetic analysis in excel. Population genetic software for teaching and research. Mol Ecol Notes 2006; 6: 288-95.

[15] Taberlet P, Luikart G. Non-invasive genetic sampling and individual identification. Biol J Linn Soc 1999; 68: 41-55.

[16] Bernet GP, Bramardi S, Calvache D, et al. Applicability of molecular markers in the context of protection of new varieties of cucumber. Plant Breed 2003; 122: 146-52. 